\title{
Metallic stripes: Separation of spin, charge, and string fluctuation
}

\author{
J. Zaanen, O. Y. Osman, and W. van Saarloos \\ Instituut Lorentz, Leiden University, P.O. Box 9506, NL-2300 RA Leiden, The Netherlands
}

(Received 6 August 1998)

\begin{abstract}
Inspired by the cuprate stripes, we consider the problem of a one-dimensional metal living on a delocalized trajectory in two dimensional space: the metallic lattice string. A model is constructed with maximal coupling between longitudinal and transversal charge motions, which nevertheless renormalizes into a minimal generalization of the Luttinger liquid: an independent set of string modes has to be added to the long-wavelength theory, with a dynamics governed by the quantum sine-Gordon model. [S0163-1829(98)51742-0]
\end{abstract}

Evidence is accumulating that the superconducting state in the cuprates is closely related to the stripe phase, where the active charge degrees of freedom are confined on antiphase boundaries in the antiferromagnetic background. ${ }^{1}$ One may argue that the stripes might be internally like onedimensional (1D) metals. Several theoretical works have appeared taking this "self-organized", one dimensionality as a starting point. $^{2}$ However, compared to conventional onedimensional metals, one has to account for the possibility that the trajectory on which the metal lives is itself delocalized in space, obviously so because of the absence of static striped order in either the superconducting or normal states. The question arises as to what can be said about the general nature of a quantum string which is internally a metal. According to the Luttinger liquid theory of 1D metallicity, all that matters at long wavelengths are the collective charge and spin oscillations which are governed by quantum sineGordon field theories (QSG). ${ }^{3}$ It can be argued that the strings of relevance to cuprates are governed by QSG as well. ${ }^{4}$ Here we will demonstrate that a fixed-point theory exists which is a minimal generalization of the Luttinger liquid: a metallic string can be like a Luttinger liquid, except that a set of string modes has to be added for the theory to be complete. Our construction rests on the assumption that a reference string state exists which is at the same time localized in space and internally charge incompressible due to a charge-density wave (CDW) instability. The CDW solitons emerging under doping make the string position fluctuate and the resulting charged kink gas maps on a spin-full fermion problem with a Luttinger liquid long-wavelength regime.

For cuprates, it appears that the state at $x=1 / 8$ corresponds with internally insulating, localized stripes. Since the average stripe separation $d$ decreases like $1 / x$ for $x \leqslant 1 / 8,{ }^{5}$ the stripes are likely internally charge incompressible. For $x>1 / 8, d$ becomes approximately $x$ independent, suggesting that the stripes are "doped" with the additional holes. At the same time, the static stripe phase shows a special stability at $x=1 / 8$; this indicates a tendency towards localization on the single stripe level. For modeling purposes we assume the electronic system on the stripe to be dominated by shortrange repulsive interactions, favoring a $4 k_{F} \mathrm{CDW}$ instability as suggested by Nayak and Wilczek ${ }^{6}$ [Fig. 1(a)]-the other possibilities are more complicated, but not necessarily qualitatively different in the present context. Finally, it is assumed that spin separates at the very beginning and can be ignored all along. Since the stripe sweeps through a spin-full background, the neglect of spin is certainly not justifiable, and further work is needed on this fascinating problem.

If the string does not delocalize, the remaining problem of a doped $4 k_{F}$ charge density wave is well understood. ${ }^{7,8} \mathrm{~A}$ representative model, in the sense of adiabatic continuity, is the extended Hubbard model with both $U$ (on-site repulsion) and $V$ (nearest-neighbor repulsion) large compared to the bandwidth. At low doping, lattice commensuration dominates and the relevant lattice scale physics is that of solitons. Using simple kinematics, Kivelson and Schrieffer $^{9}$ pointed out that the injected hole splits into propagating soliton and antisoliton excitations, both carrying half the charge of the hole [Fig. 1(b)]. The soliton dynamics is described in terms of a spinless fermion problem:

$$
H_{\mathrm{CDW}}=\sum_{i j} t^{\prime}(i j) c_{i}^{\dagger} c_{j}+\sum_{i j} V^{\prime}(i j) n_{i} n_{j}
$$

Each soliton, created by $c^{\dagger}$, is subject to short-range hoppings $\left(t^{\prime}\right)$ and (repulsive) interactions $\left(V^{\prime}\right)$. This problem is dual to a bosonic quantum sine-Gordon theory:

$$
H_{\rho, \text { ren }}=\frac{v_{\rho}}{2} \int d x\left[K_{\rho} \Pi_{\rho}^{2}+\frac{1}{K_{\rho}}\left(\partial_{x} \phi_{\rho}\right)^{2}+g \sin \left(\alpha \phi_{\rho}\right)\right],
$$

where $v_{\rho}$ and $K_{\rho}$ correspond with the charge velocity and charge stiffness, respectively. Away from quarter filling, this theory is in the weak-coupling regime (the sine interaction is an irrelevant operator) and the long-wavelength dynamics is governed by free field theory (Luttinger liquid), completely specified by the renormalized stiffness and velocity. These parameters have to be calculated numerically, and their behavior is well documented for the extended Hubbard model. ${ }^{7,8}$

The most elementary physical interpretation of the quantum sine-Gordon model, Eq. (2), actually corresponds with a free string moving on a lattice: the field $\phi$ is the transversal displacement $[z(l)]$ at point $l$ of the string, while the cosine term describes the lattice washboard on which the string moves $\left[\alpha \phi_{c} \rightarrow 2 \pi z(l) / a\right]$. The weak- and strong-coupling limits are easily understood as a freely meandering string and one which is fully localized due to the lattice potential. As was discussed elsewhere in detail, ${ }^{4}$ this notion is of relevance 
(a)

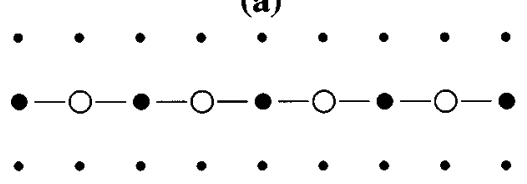

(b)

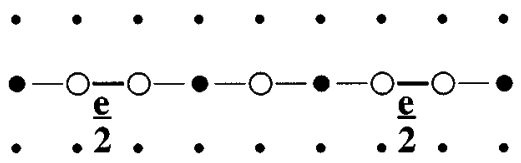

(c)

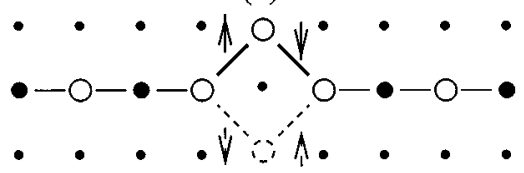

(d)

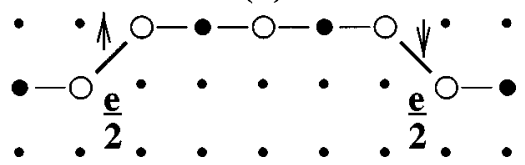

FIG. 1. Soliton dynamics in a strongly coupled doped $4 k_{F}$ stripe. (a) The reference state: localized stripe with $4 k_{F}$ chargedensity wave. (b) If the stripe is rigid, the doped hole separates in a left- and right-moving soliton, both carrying half the electron charge. (c) When the curvature energy becomes less than the charge compressibility energy, the hole can escape "sideways." (d) As a result, the solitons now carry a transversal (step up/down) flavor, which is like a spin degree of freedom. Holes tunneling through the stripe lead to fluctuations in the transversal flavor, see (c).

in the context of transversally fluctuating insulating stripes. In analogy with the charge-density wave problem, the relevant lattice scale dynamics is that of transversal solitons or "kinks." Consider the vicinity of the string delocalization transition. Because the lattice potential dominates, the microscopic configurations tend to be those of Fig. 1(d), where the string is localized on a particular lattice row $n$, and the exceptions are where the string jumps to neighboring rows $n$ \pm 1 . The origin of the collective motions of the string lies in the microscopic dynamics of these kink excitations. The tightly localized kinks of Fig. 1 are assumed to be a legitimate starting point to discuss string fluctuations, in the sense that they are connected by adiabatic continuation to more realistic string microscopies.

The existence of a localized stripe with internal $4 k_{F}$ density wave allows for a simple unification of the microscopic string and internal charge dynamics. Obviously, the fixed 1D electron trajectory assumed in the Luttinger liquid is no longer a given for electronic stripes. For a fixed trajectory, it costs an energy equal to the jump in the thermodynamic potential $\delta \mu$ to dope the charge-density wave with an additional carrier. On the stripe, this ("longitudinal") energy cost can be reduced by letting the charge escape "sideways," causing a transversal displacement, at the expense of paying a curvature energy. Hence, when this curvature energy becomes less than the energy cost associated with compressing the charge, the doped holes will "carry a string fluctuation." In terms of the strong coupling kinks/solitons, the microscopic mechanism of transversal relaxation is obvious: the doped hole corresponds with a double kink in the string which is at the same time a soliton-antisoliton pair in the on-string charge-density wave-see Fig. 1. Starting from the CDW/localized string reference state, the kinks and the solitons are the same objects. This scenario corresponds with the strongest possible microscopic coupling between the onstring metallicity and the string fluctuation. Due to the string fluctuation, the CDW solitons acquire a transversal flavor: the (anti) soliton can move the string either in an upward ( $\uparrow$ ) or downward $(\downarrow)$ direction [Figs. 1(c) and 1(d)]. This transversal freedom is like a $s=1 / 2$ isospin degree of freedom. Since the CDW solitons can be described in terms of spinless fermions, the string soliton dynamics relates to a spinful fermion system. Since the string dynamics is like the spin dynamics in a standard 1D metal, it follows that the separation of charge and string dynamics is generic.

The qualitative nature of the long-wavelength physics can be inferred from the strong coupling cartoon of Fig. 1, leaving the nonuniversal parameters of the theory to be determined from a more realistic microscopic theory. We seek a generalization of the spinless fermion model, incorporating the string flavor in terms of isospin labels $\uparrow$ and $\downarrow$ attached to the fermions [see Figs. 1(c) and 1(d)]. As a first guess, one could take the spin-full version of Eq. (1) with a hard-core $(U \rightarrow \infty)$ condition: the string flavor is conserved under the hopping of the solitons. However, this neglects the specifics of the transversal sector: (i) curvature energy is associated with the order of the isospins. Obviously a $\uparrow \downarrow$ isospin configuration of neighboring solitons involves a different curvature energy than parallel configurations. These curvature energies can be absorbed in isospin-only Ising terms $\sim S_{i}^{z} S_{j}^{z}\left[\vec{S}_{i}=\Sigma_{\alpha \beta} c_{i \alpha}^{\dagger}(\vec{\sigma})_{\alpha \beta} c_{i \beta}\right]$. (ii) The overall transversal string displacement $u$ after arclength $r$ becomes $\left(a_{0}\right.$ is the lattice constant)

$$
u(r)-u(0)=a_{0} \int_{0}^{r} d x \sigma^{z}(x)
$$

where $\vec{\sigma}(x)=\Sigma_{m} \vec{S}_{n} \delta\left(x-x_{m}\right)\left(x_{m}\right.$ is the position of the $m$ th kink). As long as this quantity is conserved the string remains localized. For $U \rightarrow \infty$ there is no kinetic exchange, and Ising isospin terms do not cause fluctuations in $u(l)$ either. In order to make the string displacement fluctuate, the isospins should be exchanged and this is possible if and only if two kinks recombine into a hole, because the hole can tunnel through the string, see Fig. 1(c). The simplicity of the argument is deceptive: this is an explicit realization of the idea of topological confinement. ${ }^{10}$ Because of their topological nature, the kinks are strictly limited to the 1D string trajectory. In order to sweep the string through 2D space, the kinks have to pair up in holes, because the latter can propagate in $2 \mathrm{D}$.

In isospin language, the hole tunneling corresponds with spin-flip $(X Y)$ terms $\sim S_{i}^{+} S_{j}^{-}+$H.c. Notice that the energy barrier involves the difference in curvature energy and the charge-compression energy. This might well be a small number, and the hole-tunneling rate can in principle be large. Assuming everything to be short ranged, we arrive at the following model, in standard notation $\left(n=n_{\uparrow}+n_{\downarrow}\right)$ : 


$$
\begin{aligned}
H_{0}^{s t r}= & -t \sum_{n \sigma}\left(c_{n+1 \sigma}^{\dagger} c_{n \sigma}+c_{n \sigma}^{\dagger} c_{n+1, \sigma}\right)+V \sum_{n} n_{n} n_{n+1} \\
& +U \sum_{n} n_{n \uparrow} n_{n \downarrow}+J_{\|} \sum_{\langle n m\rangle} S_{n}^{z} S_{m}^{z} \\
& +\frac{J_{\perp}}{2} \sum_{\langle n m\rangle}\left(S_{n}^{+} S_{m}^{-}+S_{n}^{-} S_{m}^{+}\right) .
\end{aligned}
$$

$U$ should be taken to infinity, while $t, V, J_{\|}$, and $J_{\perp}$ parametrize the kink kinetic energy, the "string neutral" kink repulsion, the curvature energy, and the hole tunneling rate, respectively.

Although we are not aware of explicit calculations on this particular model, the structure of the long-wavelength dynamics can be deduced directly from the work by Luther and Emery $^{11}$ (see also Ref. 4). When $J_{\|}=J_{\perp}$, Eq. (4) is like the extended Hubbard model with finite $U$, at a low carrier density. The general case $J_{\|} \neq J_{\perp}$ corresponds with an interacting electron system with a spin-orbit coupling causing uniaxial spin anisotropy. Charge and string flavor will separate always and the charge dynamics is described by the QSG model, Eq. (2). Away from the quarter-filled point, Umklapp scattering becomes irrelevant and the charge dynamics at long wavelength is described by free fields characterized by the fully renormalized charge velocity and stiffness $\widetilde{v}_{\rho}$ and $\widetilde{K}_{\rho}$ which will behave similarly to the ones of the extended Hubbard model in the strongly coupled regime.

A crucial observation is that the gross behavior in the string sector is determined by the "isospin-only" problem. The isospin dependencies of the interactions are explicit in Eq. (4), and the isospin-only problem is nothing else but a $X X Z$ problem with $S=1 / 2$, which was solved a long time ago. ${ }^{12}$ If $-1<J_{\|} / J_{\perp}<1$, the Ising interaction is irrelevant and the system falls in the $X Y$ universality class, as described by free field theory-the free string is recovered. When $\left|J_{\|}\right|>\left|J_{\perp}\right|$ the Ising anisotropy takes over and the string modes acquire a mass-metallicity is a necessary but insufficient condition for the string delocalization. Physically, strings in this regime are of the "disordered flat", variety. ${ }^{4}$ Although kinks proliferate and delocalize, their internal string flavor (isospin) is ordered, as a compromise between kinetic energy and lattice commensuration energy. The "ferromagnetic"' case $\left(J_{\|}<\left|J_{\perp}\right|\right)$ corresponds with a "slanted", phase: ${ }^{4}$ the string is still localized, but it takes some direction in space determined by the density of kinks. For $J_{\|}>\left|J_{\perp}\right|$ the string is on average bond centered; this phase is related to the hidden order present in Haldane spin chains. $^{4}$

The most interesting phase is the delocalized string, and we will now show that the asymptotic structure of Luttinger liquid theory implies a rather weak influence of the string metallicity on the string fluctuation. A quantity of physical interest is the mean-square transversal displacement of the string, ${ }^{13}$ using Eq. (3):

$$
\left\langle[u(r)-u(0)]^{2}\right\rangle=a_{0}^{2} \int_{0}^{r} d x d x^{\prime}\left\langle\sigma^{z}(x) \sigma^{z}\left(x^{\prime}\right)\right\rangle .
$$

The spin-spin correlation function of a one-dimensional metal has the asymptote ( $K_{\sigma}$ is the spin stiffness)

$$
\left\langle\sigma^{z}(x) \sigma^{z}(0)\right\rangle=\frac{C_{1}}{x^{2}}+\frac{C_{2} \cos \left(2 k_{F} x\right)}{|x|^{\eta}},
$$

where $\eta=K_{\sigma}+K_{\rho}$. Although $\eta \geqslant 1, \eta$ can be less than 2; in this case the staggered component of the spin-spin correlator could become important for the string correlator, Eq. (5). However, it is easy to see that in the additional integrations in Eq. (5) the staggered component behaves as if it falls off by one power more than $\eta \quad\left[\int d x \cos \left(2 k_{f} x\right) / x^{\eta}\right.$ $\left.\rightarrow \int d x 1 / x^{\eta+1}\right]$. Since $\eta \geqslant 1$ it follows that the large $r$ asymptote of Eq. (5) is governed by the uniform component $\sim C_{1}$ in Eq. (6). Using that $\int_{0}^{r} d x d x^{\prime} f\left(x-x^{\prime}\right)=\int_{-r}^{r}(2 r$ $-|x|) f(x)$ and the fact that $\int_{-\infty}^{\infty} d x\left\langle\sigma^{z}(x) \sigma^{z}(0)\right\rangle=0$ it follows that the metallic string behaves asymptotically as a free string, 13

$$
\left\langle[u(r)-u(0)]^{2}\right\rangle=-2 a_{0}^{2} C_{1} \ln \left(r / r_{c}\right)+\text { const }
$$

with a constant coming from short-wavelength physics and where $r_{c}$ is a microscopic cutoff.

Although not often discussed, the amplitude $C_{1}$ of the uniform component of the spin-spin correlation is also in the metal entirely determined by the spin sector, which implies in the present context that the strength of the string fluctuation is determined primarily by the transversal sector. This can be easily understood from the insight by Schulz ${ }^{14}$ that the charge sector of the Luttinger liquids is nothing else than a 1D harmonic ("floating") Wigner crystal of (in our case) solitons. To every soliton a spin is attached and Schulz shows that by factorizing $\left\langle\sigma^{z}(z) \sigma^{z}(0)\right\rangle$ in a spin and a charge correlator and by treating the charge sector on the Gaussian level, it follows that the exponent $\eta$ in the staggered component of Eq. (6) is the sum of the charge and spin stiffnesses $K_{\sigma}$ and $K_{\rho}$ because the spin system "rides" on the harmonically fluctuating charge solid. Following the same alley, it is straightforward to show that this charge fluctuation is invisible in the uniform correlations responsible for the string delocalization.

We are now in the position to completely quantify Eq. (7). Using Haldane's expressions for the Luttinger liquid correlation functions ${ }^{15}$ and realizing that the cutoff $r_{c}$ corresponds with the lattice constant $a$ of the soliton Wigner crystal, we get

$$
\left\langle[u(r)-u(0)]^{2}\right\rangle=\frac{a_{0}^{2} K_{\sigma}}{2 \pi^{2}} \ln (r / a)+\text { const. }
$$

Let us now assume that the above model applies literally to cuprates. Assuming that finite range string-string interactions are unimportant, a measure for the importance of the single string quantum fluctuations is the quantum collision length $\xi_{c}$, obtained by demanding that the rms displacement of a string becomes of order of the mean string-string separation $d\left(\simeq 4 a_{0}\right)::^{13}\left\langle\left[u\left(\xi_{c}\right)-u(0)\right]^{2}\right\rangle=d^{2}$. Using the soliton lattice constant $a=a_{0} /(8 x-1)$ in terms of the doping density $x$, together with the expression for the spin stiffness ${ }^{12} K_{\sigma}^{-1}$ $=1 / 2+(1 / \pi) \arcsin \left(J_{\|} / J_{\perp}\right)$, we obtain

$$
\xi_{c}=\frac{a_{0}}{8 x-1} e^{\left(d / a_{0}\right)^{2}\left[\pi^{2}+2 \pi \arcsin \left(J_{\|} / J_{\perp}\right)\right]} .
$$


The doping density only enters in the prefactor via the trivial soliton-lattice constant rescaling, while $\xi_{c}$ depends exponentially on the stripe separation and the transversal scales. Hence, the metallicity induced long-wavelength string fluctuations can only play a decisive role in the quantum melting of the stripe phase if the factor in the exponent becomes of order unity. Because of the various numerical factors, this only happens if the string sector is very close to the "ferromagnetic" point $J_{\|} / J_{\perp} \rightarrow-1$. It appears as very unlikely that such a fine tuning occurs in cuprates so we conclude that on-string metallicity is not an important factor for the quantum melting of the stripe phase.

In summary, we have addressed the problem of a lattice string which is internally a metal. Starting from specific mi- croscopic assumption inspired by cuprate stripes, we have shown that its long-wavelength dynamics is a straightforward generalization of the Luttinger liquid where the usual theory has to be extended with a sector of transversal sound modes. Although intended as a demonstration of the existence of a fixed point (with probably a finite basin of attraction), a literal interpretation of the microscopic model shows that the string fluctuation is quite insensitive to the internal metallicity. As applied to cuprates, this observation offers a rationale for the surprising insensitivity of the static stripe phases in, e.g., Luttinger liquid theory materials against stripe doping.

Important discussions are acknowledged with H. J. Eskes in an early stage of this research.
${ }^{1}$ J. M. Tranquada et al., Phys. Rev. Lett. 78, 338 (1997); J. M. Tranquada, Physica B 241-243, 745 (1998), and references therein.

${ }^{2}$ V. J. Emery, S. A. Kivelson, and O. Zachar, Phys. Rev. B 56, 6120 (1997); Yu. A. Krotov, D.-H. Lee, and A. V. Balatsky, ibid. 56, 8367 (1997); S. A. Kivelson, E. Fradkin, and V. J. Emery, Nature (London) 393, 550 (1998); J. Zaanen, cond-mat/9711009, J. Phys. Chem. Solids (to be published).

${ }^{3}$ J. Voit, Rep. Prog. Phys. 57, 977 (1994), and references therein.

${ }^{4}$ H. Eskes et al., Phys. Rev. B 54, R724 (1996); 58, 6963 (1998);

C. Morais-Smith et al., ibid. 58, 1 (1998).

${ }^{5}$ K. Yamada et al., Physica C 282-287, 85 (1997).

${ }^{6}$ Y. Nayak and F. Wilczek, Phys. Rev. Lett. 78, 2465 (1997).

${ }^{7}$ F. D. M. Haldane, Phys. Rev. Lett. 45, 1358 (1980); M. P. M. den
Nijs, Phys. Rev. B 23, 6111 (1981); V. J. Emery, Phys. Rev. Lett. 65, 1076 (1990).

${ }^{8}$ K. Penc and F. Mila, Phys. Rev. B 49, 9670 (1994); K. Sano and Y. Ono, J. Phys. Soc. Jpn. 63, 1250 (1994).

${ }^{9}$ S. A. Kivelson and J. R. Schrieffer, Phys. Rev. B 25, 6447 (1982); J. R. Schrieffer, Proc. Int. School. Phys. Enrico Fermi LXXXIX, edited by F. Bassani (Elsevier, New York, 1985).

${ }^{10}$ S. Chakravarty and P. W. Anderson, Phys. Rev. Lett. 72, 3859 (1991).

${ }^{11}$ A. Luther and V. J. Emery, Phys. Rev. Lett. 33, 589 (1974).

${ }^{12}$ A. Luther and I. Peschel, Phys. Rev. B 12, 3908 (1975).

${ }^{13}$ J. Zaanen, M. Horbach, and W. van Saarloos, Phys. Rev. B 53, 8671 (1996).

${ }^{14}$ H. J. Schulz, in Correlated Electron Systems, edited by V. J. Emery (World Scientific, Singapore, 1993).

${ }^{15}$ F. D. M. Haldane, Phys. Rev. Lett. 47, 1840 (1981). 